\title{
Bintrafusp Alfa, a Bifunctional Fusion Protein Targeting TGF $\beta$ and PD-L1, in Patients with Esophageal Squamous Cell Carcinoma: Results from a Phase 1 Cohort in Asia
}

\author{
Chia-Chi Lin ${ }^{1} \cdot$ Toshihiko Doi $^{2} \cdot$ Kei Muro ${ }^{3}$ Ming-Mo Hou ${ }^{4} \cdot$ Taito Esaki $^{5} \cdot$ Hiroki Hara $^{6} \cdot$ Hyun Cheol Chung $^{7}$. \\ Christoph Helwig $^{8} \cdot$ Isabelle Dussault $^{9,10} \cdot$ Motonobu Osada ${ }^{11,12} \cdot$ Shunsuke Kondo ${ }^{13}$ (i)
}

Accepted: 19 March 2021 / Published online: 11 April 2021

(c) The Author(s) 2021

\begin{abstract}
Background Patients with esophageal squamous cell carcinoma (SCC) have limited treatment options. Blocking transforming growth factor- $\beta$ (TGF $\beta$ ), which can be overexpressed in these tumors, may enhance responses to programmed cell death protein 1/programmed death-ligand 1 [PD-(L)1] inhibitors. Bintrafusp alfa is a first-in-class bifunctional fusion protein composed of the extracellular domain of the TGF $\beta$ receptor II (TGF $\beta$ RII) (a TGF $\beta$ "trap") fused to a human IgG1 monoclonal antibody blocking PD-L1.

Objective The objective of this study was to investigate the safety and efficacy of bintrafusp alfa in Asian patients with pretreated, PD-L1-unselected esophageal SCC.

Patients and Methods In a phase 1 study, Asian patients with pretreated esophageal SCC received bintrafusp alfa $1200 \mathrm{mg}$ every 2 weeks until disease progression, unacceptable toxicity, or withdrawal. The primary endpoint was safety/tolerability with a goal of exploring clinical activity.

Results By the database cutoff of August 24, 2018, 30 patients (76.7\% had two or more prior anticancer regimens) received bintrafusp alfa for a median of 6.1 weeks; two remained on treatment. Nineteen patients $(63.3 \%)$ had treatment-related adverse events, seven (23.3\%) with grade $3 / 4$ events, and there were no treatment-related deaths. The confirmed objective response rate (ORR) per independent review was 10.0\% (95\% confidence interval [CI] 2.1-26.5); responses lasted 2.8-8.3 + months. All responses occurred in immune-excluded tumors. Investigator-assessed confirmed ORR was $20.0 \%$ (95\% CI 7.7-38.6). Median overall survival was 11.9 months (95\% CI 5.7-not reached).

Conclusions Bintrafusp alfa demonstrated a manageable safety profile and efficacy in Asian patients with pretreated esophageal SCC.

Clinical Trials Registration NCT02699515.
\end{abstract}

Shunsuke Kondo

shkondo@ncc.go.jp

Extended author information available on the last page of the article 


\section{Key Points}

Bintrafusp alfa is a first-in-class bifunctional fusion protein that was designed for colocalized, simultaneous inhibition of two nonredundant immunosuppressive pathways, transforming growth factor- $\beta$ (TGF $\beta)$ and programmed death-ligand 1 (PD-L1), within the tumor microenvironment.

In this expansion cohort of a phase 1 study, bintrafusp alfa had a manageable safety profile and demonstrated clinical activity in Asian patients with pretreated, PD-L1-unselected esophageal squamous cell carcinoma.

Along with the results of an expansion cohort of patients with advanced, post-platinum esophageal adenocarcinoma from a separate phase 1 study described in the accompanying article, further investigation of bintrafusp alfa in esophageal cancer is warranted.

\section{Background}

Globally, esophageal cancer is one of the most common cancer types, causing more than 500,000 deaths in 2018 [1]. The prognosis is poor for patients with esophageal cancer, and the age-standardized 5-year survival rates range from 10 to $30 \%$ after correction for background mortality [2]. Additionally, the majority of patients have advanced disease at diagnosis, with 5-year survival rates below $10 \%$ [3].

The incidence of esophageal cancers varies geographically, with eastern Asia having the highest regional incidence in the world [1]. Furthermore, the histologic subtypes of esophageal cancer, squamous cell carcinoma (SCC) and adenocarcinoma (AC), also vary by region and etiology. Esophageal SCC accounts for approximately $90 \%$ of all esophageal cancer cases, most of which occur in China [1, 4]. Other Asian countries also have high rates of esophageal SCC, with an increasing incidence in Taiwan and Japan [4-6]. Tobacco use, alcohol consumption, drinking hot mate tea, chewing betel quid, and human papillomavirus infection have all been suggested to increase the risk of esophageal SCC, which is most common in males [4, 7].

Genetic analysis of esophageal SCC has identified recurring alterations in CCND1, TP53, SOX2, and TP63 within cancer cells that can potentially impact disease development and progression [8]. Other factors can act outside of tumor cells in the tumor microenvironment (TME) to promote disease progression [9]. Transforming growth factor- $\beta$ (TGF $\beta$ ) is a cytokine that can impact a wide array of functions in the TME and tumor cells $[10,11]$. TGF $\beta$ signaling is complex, often acting as a tumor suppressive pathway in early stages of disease across cancers, including esophageal SCC $[11,12]$. However, in later stages of disease, TGF $\beta$ can have a tumor-promoting role and be upregulated in advanced esophageal SCC [11-15]. The TGF $\beta$ pathway can promote cancer progression and immune evasion in the TME via regulatory effects on immune cells, and may impact processes such as angiogenesis and epithelial-to-mesenchymal transition $[10,16]$. Stimulation with recombinant TGF $\beta 1$ in preclinical studies led to an increase in mesenchymal markers and invasion capabilities of esophageal SCC cell lines [17].

Unlike in patients with esophageal AC, targeted therapies were not historically recommended for patients with esophageal SCC. Treatment was limited to standard chemotherapy, with a median overall survival $(\mathrm{OS})<1$ year for patients with pretreated esophageal SCC [18-22]. Immune checkpoint inhibitors have offered a new therapeutic approach for esophageal SCC. Results of the global phase $3 \mathrm{KEY}$ NOTE-181 trial showed that second-line treatment with pembrolizumab improved median OS compared with chemotherapy in a subgroup of patients with esophageal SCC tumors expressing programmed death-ligand 1 (PD-L1) (combined positive score $[\mathrm{CPS}] \geq 10 ; 10.3$ vs 6.7 months) [23]. An improvement in median OS was also observed with second-line pembrolizumab treatment in an extension of this study in Chinese patients with tumors expressing PD-L1 (12.0 vs 5.4 months) [24]. A similar trend was reported in the phase 2 KEYNOTE-180 study of pembrolizumab in patients with PD-L1-positive esophageal SCC that progressed after two or more lines of systemic therapy, in which treatment with pembrolizumab resulted in a high objective response rate (ORR) and long duration of response [25, 26]. These studies led to regulatory approvals of pembrolizumab for patients who had PD-L1-positive (CPS $\geq 10$ ) esophageal SCC with disease progression after one prior line of therapy in China and one or more prior lines of therapy in the United States [24, 25]. Of note, interim analysis of the global phase 3 KEYNOTE-590 trial demonstrated that first-line treatment with pembrolizumab plus chemotherapy (fluorouracil plus cisplatin) improved median OS versus chemotherapy (12.6 vs 9.8 months) [27], which has resulted in priority review for pembrolizumab in the first-line treatment of patients with locally advanced unresectable or metastatic esophageal cancer [28]. Findings from the ATTRACTION-3 study, which evaluated second-line nivolumab treatment in esophageal SCC, showed a 2.5-month improvement in median OS when compared with chemotherapy (10.9 vs 8.4 months) [29]. These results led to the approval of nivolumab in Japan and the United States for unresectable, advanced or recurrent esophageal cancer that progressed following chemotherapy. However, the overall efficacy of programmed cell death protein 1 (PD-1) therapies in patients who progressed following 
chemotherapy and regardless of PD-L1 status was limited; ORRs ranged from 14 to $19 \%$, and the median OS ranged from 6.8 to 10.9 months $[23,26,29,30]$.

Interestingly, multiple preclinical studies have shown that blocking TGF $\beta$ can enhance the antitumor effect of PD-(L) 1 inhibitors and that the combined use of a TGF $\beta$ blocker and an anti-PD-(L)1 agent improve efficacy over either agent alone in mouse models [31-34]. Bintrafusp alfa is a first-in-class bifunctional fusion protein composed of the extracellular domain of the human TGF $\beta$ receptor II (TGF $\beta$ RII or TGF $\beta$ "trap") fused via a flexible linker to the C-terminus of each heavy chain of the IgG1 antibody blocking PD-L1 (anti-PD-L1) [35]. Bintrafusp alfa is designed for colocalized, simultaneous inhibition of two nonredundant immunosuppressive pathways (TGF $\beta$ and PD-L1) within the TME [35]. This may provide an enhanced treatment effect, potentially improving clinical benefit compared with anti-PD-(L)1 monotherapies [35-37].

In two phase 1 studies (NCT02517398 and NCT02699515), bintrafusp alfa had a manageable safety profile and preliminary signs of clinical efficacy in patients with heavily pretreated advanced solid tumors [38-40]. In this report, we present results from an expansion cohort of a phase 1 study with the objective of investigating the safety and efficacy of bintrafusp alfa in Asian patients with pretreated, PD-L1-unselected esophageal SCC.

\section{Patients and Methods}

\subsection{Study Design and Participants}

NCT02699515 is a phase 1, open-label study that investigated the safety and efficacy of bintrafusp alfa in Asian patients with heavily pretreated solid tumors, and included multiple expansion cohorts in specific tumor types. This work describes the esophageal SCC expansion cohort, which enrolled patients with histologically or cytologically confirmed disease for whom standard therapy did not exist or had failed. Eligible patients were aged 20 years or older with measurable disease by Response Evaluation Criteria in Solid Tumors version 1.1 (RECIST 1.1) and had an Eastern Cooperative Oncology Group (ECOG) performance status of 0 or 1 , a life expectancy of $\geq 12$ weeks, and adequate renal, hepatic, and hematological function. Additionally, the availability of tumor (primary or metastatic) archival material or fresh biopsies taken within 28 days before the first administration of bintrafusp alfa was required for eligibility. Patients were not selected based on PD-L1 expression or other biomarkers. Patients with a history of central nervous system metastases or who had received prior therapy with TGF $\beta(R)$-targeting agents or immune checkpoint inhibitors were ineligible for this study.

\subsection{Treatment and Assessments}

Patients received bintrafusp alfa at the recommended phase 2 dose of $1200 \mathrm{mg}$ via intravenous infusion over $1 \mathrm{~h}$ once every 2 weeks for up to 12 months until confirmed disease progression, unacceptable toxicity, or trial withdrawal [41]. A flat dose of $1200 \mathrm{mg}$ every 2 weeks was selected as the recommended phase 2 dose of bintrafusp alfa based on safety/tolerability, pharmacokinetic, and pharmacodynamic data, as well as preliminary population pharmacokinetic and exposure-response modeling of phase 1 data [41, 42]. Additional treatment beyond 12 months could be permissible if the investigator believed a patient may benefit. Dose reductions were not allowed. Responses were assessed by imaging every 6 weeks during the treatment period and every 12 weeks following treatment until disease progression. Additional scans were performed to confirm all responses, and disease progression was confirmed by additional scans. An independent review committee reviewed all radiographic images and performed a blinded determination as to whether the criteria for tumor response or progression according to RECIST 1.1 had been met. Safety assessments, such as performance status evaluation, physical examination, and clinical laboratory tests, were performed routinely throughout the study, and adverse events (AEs) were documented at every visit. An additional safety follow-up visit was planned 28 days after the last study dose or before the start of a new treatment (whichever occurred first). Safety continued to be evaluated 10 weeks after treatment. AEs were classified and graded with the National Cancer Institute Common Terminology Criteria for Adverse Events (NCI-CTCAE) version 4.03. Any AE that was believed to be a potential immunerelated or potential TGF $\beta$-related event was considered an $\mathrm{AE}$ of special interest. A list of preselected terms from the Medical Dictionary for Regulatory Activities version 21.0 was used to identify immune-related AEs (irAEs).

\subsection{Endpoints}

The safety and tolerability of bintrafusp alfa were the primary endpoint for this expansion cohort. Key secondary endpoints were confirmed best overall response according to RECIST 1.1, duration of response, disease control rate (DCR), progression-free survival (PFS), and OS. Exploratory endpoints included the evaluation of potential predictive markers in tumors, including PD-L1 expression and immune phenotype.

\subsection{Exploratory Endpoints}

Immunohistochemistry was used to measure PD-L1 expression of formalin-fixed, paraffin-embedded tumor tissue using an anti-PD-L1 antibody clone 73-10 (Dako PD-L1 
IHC 73-10 pharmDx; Dako, Carpinteria, CA, USA). PD-L1 expression was determined on tumor cells and on cells of the TME. The data presented here are based on the percentage of tumor cells expressing PD-L1, using a threshold of $1 \%$ to characterize tumors as either PD-L1 positive $(\geq 1 \%)$ or negative $(<1 \%)$.

RNA sequencing (RNAseq) was also performed to determine whether specific tumor characteristics correlated with response to bintrafusp alfa. RNAseq was performed by Asuragen (Austin, TX, USA) using standard protocols based on ribosomal depletion from formalin-fixed, paraffinembedded archival tumor samples. Sequencing reads were aligned against the Ensembl 75 human genome (GRCh37 February 2014) with Bowtie2 version 2.2.3 (Johns Hopkins University, Baltimore, MD, USA) [43]. Gene expression was determined using RSEM version 1.2.31 and Ensembl gene annotations. Hypothesis testing was performed by comparing RSEM-computed expected counts, and transcript-permillion values were upper-quartile normalized and log transformed for additional analysis [44]. To test whether $T G F B 1$ gene expression was higher in this cohort than others across different expansion cohorts from phase 1 studies of bintrafusp alfa (NCT02699515 and NCT02517398), limma + voom was applied, modeling expression as a function of indication [45]. Patient samples that passed quality control ( $N=537$, including 28 esophageal SCC samples) were analyzed. All genes were tested with $\geq 10$ reads in $\geq 20$ samples. We report $p$ values for differential expression adjusted for testing of all genes.

Tumor mutation count was measured with an RNAseqbased variant for which tumor RNAseq data was combined with normal germline whole-exome sequencing to identify tumor-specific mutations. Illumina HiSeq System (Illumina, San Diego, CA, USA) was used to sequence tumor samples at $2 \times 50$ to a target of $10^{8}$ read pairs. Whole-exome sequencing was performed by Expression Analysis (Research Triangle Park, NC, USA) from matched peripheral blood samples using an Agilent SureSelect Human All Exon V5 kit (Agilent Technologies, Santa Clara, CA, USA). Sequencing was done on an Illumina HiSeq System with a target of 100 $\times$ coverage. Tumor RNAseq reads were mapped to hg 19 and the Ensembl gene annotations (ensGene, University of California, Santa Cruz, USA) using RNA-STAR version 2.5.0b, and whole-exome reads were mapped to hg19 using BWA-MEM version 0.7.12 [46, 47]. Mutation calling was performed on paired BAM files using VarDictJava version 1.4.2; resulting mutations were annotated with the Ensembl Variant Effect Predictor version 85 to determine the location and type of mutation $[48,49]$. The tumor mutation count for a given patient was determined as the total count of all missense mutations discovered for that sample.

Immune phenotype was determined using immunohistochemistry (PD-L1 stain and negative control) and hematoxylin and eosin stains of patient samples. We applied an exploratory classification system; samples were classified as (1) inflamed (immune cells in direct physical contact with tumor cells), (2) immune-excluded ( $\geq 1 \%$ of the tumor stroma area populated by lymphocytes, immune cells possibly located in the immediate vicinity of tumor cells, but have not efficiently infiltrated tumor cell clusters, and very infrequent physical contact between lymphocytes and tumor cells), or (3) immune-desert ( $<1 \%$ of the tumor stroma area populated by lymphocytes, no dense immune cell infiltrates, and no contact of immune cells with tumor cells) [50-53]. A pathologist who was masked to the response data was responsible for scoring the scanned images and assigning the respective phenotype.

\subsection{Statistical Analysis}

The goal of this expansion cohort was to explore the initial clinical activity of bintrafusp alfa in esophageal SCC, which was viewed as hypothesis generating. Thirty patients were planned for this expansion cohort. This sample size was determined to obtain preliminary estimates of efficacy. Data presented here were from a follow-up analysis performed based on a data cutoff approximately 1.2 years after the last patient was enrolled. The ORR was defined as the proportion of patients achieving a confirmed best overall response of complete or partial response. A 95\% Clopper-Pearson confidence interval (CI) was calculated for the ORR. DCR was defined as the proportion of patients with a best overall response of complete response, partial response, stable disease, or non-complete response/non-progressive disease. The Kaplan-Meier method was used to assess duration of response, PFS, and OS.

\section{Results}

\subsection{Patient Demographics and Characteristics}

Between January 11, 2017, and June 22, 2017, 44 patients with esophageal SCC were screened; 30 patients from ten centers in Asia met the eligibility criteria and received bintrafusp alfa. Of these, 17 patients $(56.7 \%)$ were Japanese, ten (33.3\%) were Taiwanese, and three $(10.0 \%)$ were Korean. The median age was 60 years (range 40-80), and most had an ECOG performance status of $1(n=21$ [70.0\%]). The study population was heavily pretreated, with $76.7 \%$ of patients having received two or more prior anticancer therapy regimens and $50.0 \%$ of patients having received two or more prior lines of therapy for locally advanced or metastatic disease. Approximately half of the patients $(n=$ 14 [46.7\%]) had PD-L1-positive tumors. Detailed baseline characteristics are described in Table 1. Fifteen patients 
Table 1 Patient baseline and disease characteristics

\begin{tabular}{ll}
\hline Characteristic & $N=30$ \\
\hline Median age, years (range) & $60(40-80)$ \\
Sex, $n(\%)$ & \\
$\quad$ Male & $26(86.7)$ \\
Female & $4(13.3)$ \\
ECOG performance status, $n(\%)$ & \\
0 & $9(30.0)$ \\
1 & $21(70.0)$ \\
Number of prior anticancer therapy regimens, $n(\%)$ & \\
1 & $7(23.3)$ \\
2 & $13(43.3)$ \\
$\geq 3$ & $10(33.3)$ \\
Number of prior lines of therapy for locally advanced or & \\
metastatic disease, $n(\%)$ & \\
0 & $5(16.7)$ \\
1 & $10(33.3)$ \\
$\geq 2$ & $15(50.0)$ \\
Type of prior anticancer therapy for locally advanced or & \\
metastatic disease, $n(\%)^{\mathrm{a}}$ & \\
Cytotoxic therapy & $25(83.3)$ \\
Immunotherapy except anti-PD-(L)1 & $1(3.3)$ \\
Other & $1(3.3)$ \\
Tumor cell PD-L1 expression, $n(\%)^{\mathrm{b}}$ & $14(46.7)$ \\
Positive & $13(43.3)$ \\
Negative & $3(10.0)$ \\
\hline Not evaluable & \\
\hline
\end{tabular}

ECOG Eastern Cooperative Oncology Group, $P D-1$ programmed cell death protein $1, P D-L 1$ programmed death-ligand 1

${ }^{\text {a }}$ Patients may be included in more than 1 category

${ }^{\mathrm{b}} \mathrm{A}$ threshold of $1 \%$ was used to characterize tumors as either PD-L1 positive $(\geq 1 \%)$ or negative $(<1 \%)$ using an anti-PD-L1 antibody clone $73-10$

$(50.0 \%)$ received one or more types of subsequent anticancer treatment following bintrafusp alfa, of whom 14 patients received cytotoxic therapy.

As of August 24, 2018, 30 patients received bintrafusp alfa for a median duration of 6.1 (range 2.0-74.1) weeks. The Kaplan-Meier analysis of follow-up time since first dose was 67.5 (range 0.4-80.9) weeks. Two patients (6.7\%) remained on treatment at data cutoff. Among the patients who terminated treatment, the most common reason was disease progression $(n=21[70.0 \%])$, followed by AEs $(n=$ $4[13.3 \%])$, death $(n=2[6.7 \%])$, and withdrawal of consent $(n=1[3.3 \%])$.

\subsection{Safety}

Nineteen patients $(63.3 \%)$ reported any-grade treatmentrelated AEs (TRAEs); the most common were maculopapular $\operatorname{rash}(n=6[20.0 \%])$, hypothyroidism and rash $(n=5$ [16.7\%] each), and interstitial lung disease (ILD), keratoacanthoma (KA), and pruritus ( $n=3[10.0 \%]$ each) (Table 2$)$.
Table 2 TRAEs occurring at any grade in $\geq 5 \%$ of patients or of grade 3/4 severity, and any AEs of special interest

\begin{tabular}{|c|c|c|}
\hline \multirow[t]{2}{*}{ Preferred term, $n(\%)$} & \multicolumn{2}{|l|}{$N=30$} \\
\hline & Any grade & Grade $3 / 4$ \\
\hline \multicolumn{3}{|l|}{ TRAEs } \\
\hline TRAE & $19(63.3)$ & $7(23.3)$ \\
\hline Maculopapular rash & $6(20.0)$ & $1(3.3)$ \\
\hline Rash & $5(16.7)$ & $1(3.3)$ \\
\hline Hypothyroidism & $5(16.7)$ & 0 \\
\hline Interstitial lung disease & $3(10.0)$ & $1(3.3)^{\mathrm{a}}$ \\
\hline KA & $3(10.0)$ & 0 \\
\hline Pruritus & $3(10.0)$ & 0 \\
\hline Blood creatinine increased & $2(6.7)$ & 0 \\
\hline Eczema & $2(6.7)$ & $1(3.3)$ \\
\hline Hyperthyroidism & $2(6.7)$ & 0 \\
\hline Retinal hemorrhage & $2(6.7)$ & 0 \\
\hline Amylase increased & $1(3.3)$ & $1(3.3)$ \\
\hline Blood creatine phosphokinase increased & $1(3.3)$ & $1(3.3)^{\mathrm{b}}$ \\
\hline Lichenoid keratosis & $1(3.3)$ & $1(3.3)$ \\
\hline Lip SCC & $1(3.3)$ & $1(3.3)$ \\
\hline \multicolumn{3}{|l|}{ Any AE of special interest } \\
\hline Skin lesions ${ }^{\mathrm{c}}$ & $4(13.3)$ & $1(3.3)$ \\
\hline Any irAE & $12(40.0)^{\mathrm{d}}$ & $4(13.3)$ \\
\hline Immune-related rash & $10(33.3)$ & $2(6.7)$ \\
\hline Immune-related thyroid disorders & $3(10.0)$ & 0 \\
\hline Immune-related interstitial lung disease & $2(6.7)$ & $1(3.3)^{\mathrm{a}}$ \\
\hline Other irAEs & $1(3.3)$ & $1(3.3)$ \\
\hline
\end{tabular}

$A E$ adverse event, $i r A E$ immune-related adverse event, $K A$ keratoacanthoma, NCI-CTCAE v4.03 National Cancer Institute Common Terminology Criteria for Adverse Events version 4.03, SCC squamous cell carcinoma, TRAE treatment-related adverse event

${ }^{\text {a }}$ Grade 4; was later re-evaluated by the investigator as grade 3 pneumonia

${ }^{\mathrm{b}}$ Grade 4

${ }^{\mathrm{c}}$ Includes actinic keratosis, basal cell carcinoma, Bowen's disease, hyperkeratosis, KA, lip SCC, and SCC of skin NCI-CTCAE v4.03 preferred terms

${ }^{\mathrm{d}}$ Seven patients experienced multiple different irAEs

Grade $\geq 3$ TRAEs occurred in seven patients (23.3\%), including grade 3 amylase increased, eczema, lichenoid keratosis, lip SCC, rash, maculopapular rash $(n=1$ [3.3\%] each), and grade 4 blood creatine phosphokinase increased and $\operatorname{ILD}(n=1[3.3 \%]$ each). The patient with grade 4 blood creatine phosphokinase increase also experienced grade 3 amylase increase. The grade 4 ILD was later re-evaluated by the investigator as grade 3 pneumonia, which resolved within 2 weeks following treatment with parenteral antibiotics and steroids. No treatment-related deaths occurred. Three patients (10.0\%) discontinued bintrafusp alfa due to TRAEs, including eczema, lip SCC, and maculopapular rash. One of these patients developed an itchy rash 3 days after the most 
recent administration of bintrafusp alfa (17 days after the first dose). The rash worsened from grade 1 to 3 over the course of a week, eventually leading to permanent discontinuation of study treatment. Eczema was diagnosed, treated with oral and topical steroids, and completely resolved 4 weeks after the initial onset. Another patient developed lip SCC and, based on the decision of the investigator, permanently discontinued bintrafusp alfa. The patient was treated with surgical excision of the lesion and received prophylactic antibiotics and local tetracycline for 1 week after the procedure; the AE was considered to be resolved after this treatment. The remaining discontinuation due to TRAEs occurred in a patient who developed grade 1 maculopapular rash 6 days after their second infusion of bintrafusp alfa; no relevant medical history or concomitant medications were documented. The rash was considered to be an irAE and was treated with topical steroids. The AE increased to grade 2 , leading to permanent discontinuation of bintrafusp alfa after a total of six infusions. The patient was lost to followup, and a phone interview 6 months after discontinuation revealed that the condition had become chronic and was ongoing. irAEs occurred in 12 patients $(40.0 \%)$ (Table 2, Supplementary Table S1, see the electronic supplementary material). There were no infusion-related reactions that were assessed by the investigator as being related to bintrafusp alfa. Skin lesions that may potentially be related to TGF $\beta$ were reported in four patients (13.3\%); all of these lesions were grade $\leq 3$ (KA $[n=3$ ]; hyperkeratosis, lip SCC, and SCC of skin [ $n=1$ each], Table 2, Supplementary Table S1).

\subsection{Efficacy}

Partial responses occurred in three patients, as assessed by the independent review committee (confirmed ORR 10.0\% [95\% CI 2.1-26.5]) (Table 3, Fig. 1a). Six patients had investigator-assessed partial responses (confirmed ORR 20.0\% [95\% CI 7.7-38.6]) (Supplementary Table S2, see the electronic supplementary material). The independent review committee-assessed median duration of response was 7.0 (range 2.8-8.3+) months with one ongoing response at data cutoff (Fig. 1b). Six additional patients (20.0\%) experienced disease control, resulting in a DCR of 30.0\% (95\% CI 14.7-49.4).

The median PFS was 1.4 (95\% CI 1.3-2.8), with a 6-month PFS rate of $21.4 \%$ (95\% CI 8.7-37.8), per independent review committee assessment (Fig. 2a). Similar results were obtained by investigator read, with a median PFS of 1.4 months (95\% CI 1.3-4.2) and 6-month rates of 27.6\% (95\% CI 13.1-44.3) (Supplementary Figure S1). Median OS was 11.9 (95\% CI 5.7-not reached) months in the cohort. The OS rate was $68.6 \%$ (95\% CI 48.2-82.3) at 6 months and $46.4 \%$ (95\% CI 27.4-63.5) at 12 months (Fig. 2b).

\subsection{Biomarker Analyses}

The three partial responses by independent review committee assessment occurred in PD-L1-positive tumors; however, responses by investigator assessment were observed independently of tumor cell PD-L1 expression (Table 3). Additionally, responses occurred independently of PD-L1

Table 3 Treatment response to bintrafusp alfa $(N=30)$

\begin{tabular}{lll}
\hline & Independent review committee & Investigator \\
\hline Confirmed best overall response, $n(\%)$ & & 0 \\
Complete response & 0 & $3(10.0)$ \\
Partial response & $3(10.0)$ & $5(20.0)$ \\
Stable disease & $3(10.0)$ & 0 \\
Non-complete response/non-progressive disease & $18(60.0)$ & $17(56.7)$ \\
Progressive disease & $3(10.0)$ & $2(6.7)$ \\
Not evaluable & $3(10.0)$ & $6(20.0)$ \\
Confirmed ORR, $n(\%)$ & $2.1-26.5$ & $7.7-38.6$ \\
$95 \%$ CI & $9(30.0)$ & $11(36.7)$ \\
DCR, $n(\%)$ & $14.7-49.4$ & $(19.9-56.1)$ \\
$95 \%$ CI & $7.0(2.8-8.3+)$ & $7.0(4.2+-11.1+)$ \\
Median duration of response, months (range) & & $2 / 14(14.3)$ \\
ORR by tumor cell PD-L1 expression, $n / N(\%)^{\mathrm{a}}$ & $3 / 14(21.4)$ & $3 / 13(23.1)$ \\
Positive & $0 / 13(0)$ & $1 / 3(33.3)$ \\
Negative & $0 / 3(0)$ & \\
Not evaluable & & \\
\hline
\end{tabular}

$C I$ confidence interval, $D C R$ disease control rate, $O R R$ objective response rate, $P D-L 1$ programmed death-ligand 1

${ }^{a} A$ threshold of $1 \%$ was used to characterize tumors as either PD-L1 positive $(\geq 1 \%)$ or negative $(<1 \%)$ using an anti-PD-L1 antibody clone 73-10 

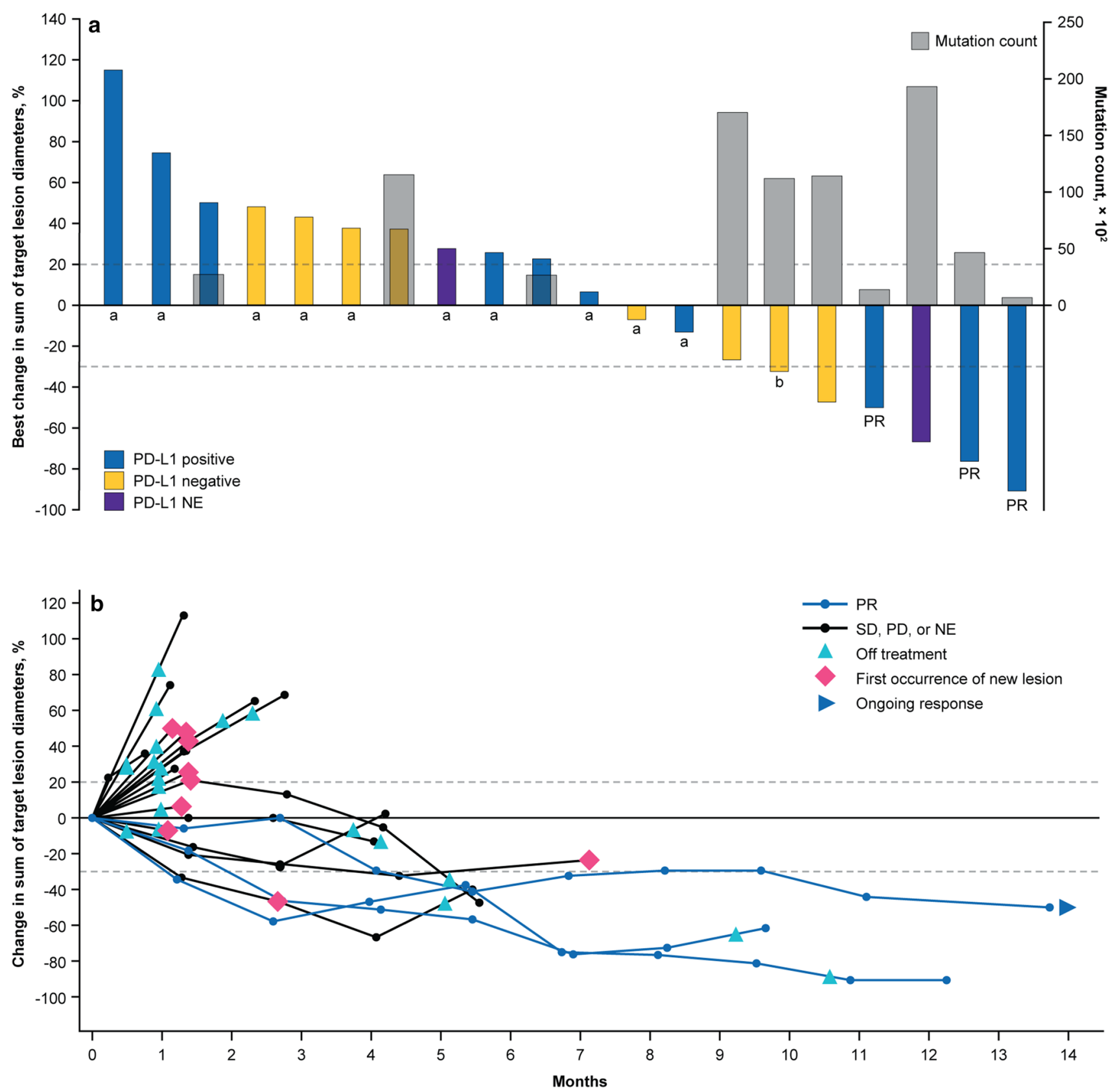

Fig. 1 Tumor response to bintrafusp alfa assessed by independent review. a Best change in sum of diameters and tumor mutation count. A threshold of $1 \%$ was used to characterize tumors as either PD-L1 positive $(\geq 1 \%)$ or negative $(<1 \%)$ using an anti-PD-L1 antibody clone 73-10. Three patients had non-evaluable PD-L1 expression. b Time to and duration of response. The upper dashed line represents progression at $20 \%$ increase in size of target lesions, and the lower dashed line represents the RECIST boundary for PR at 30\% decrease in size of target lesions. Ten patients are not shown due to having

expression in the TME, regardless of whether determined by independent review committee or investigator (data not shown). Immune phenotype analysis showed all responses occurred in tumors with an immune-excluded phenotype either no target lesions identified by independent review committee prior to the first dose $(n=6)$, no post-baseline assessment $(n=2)$, or other reasons $(n=2)$. $N E$ not evaluable, $P D$ progressive disease, $P D$ 1 programmed cell death protein $1, P D-L 1$ programmed death-ligand 1, PR partial response, RECIST Response Evaluation Criteria in Solid Tumors, $S D$ stable disease. ${ }^{\text {a }}$ Tumor mutation count unavailable. ${ }^{\mathrm{b}} \mathrm{Pa}-$ tient had a best change in sum of diameters of $>30 \%$ that did not meet the criteria for a PR at the next tumor assessment

(Fig. 3a). The ORR by independent review committee was $0 \%$ in the inflamed, $12.0 \%$ in the immune-excluded, and $0 \%$ in the immune-desert phenotypes. However, response to bintrafusp alfa was observed independently of tumor 

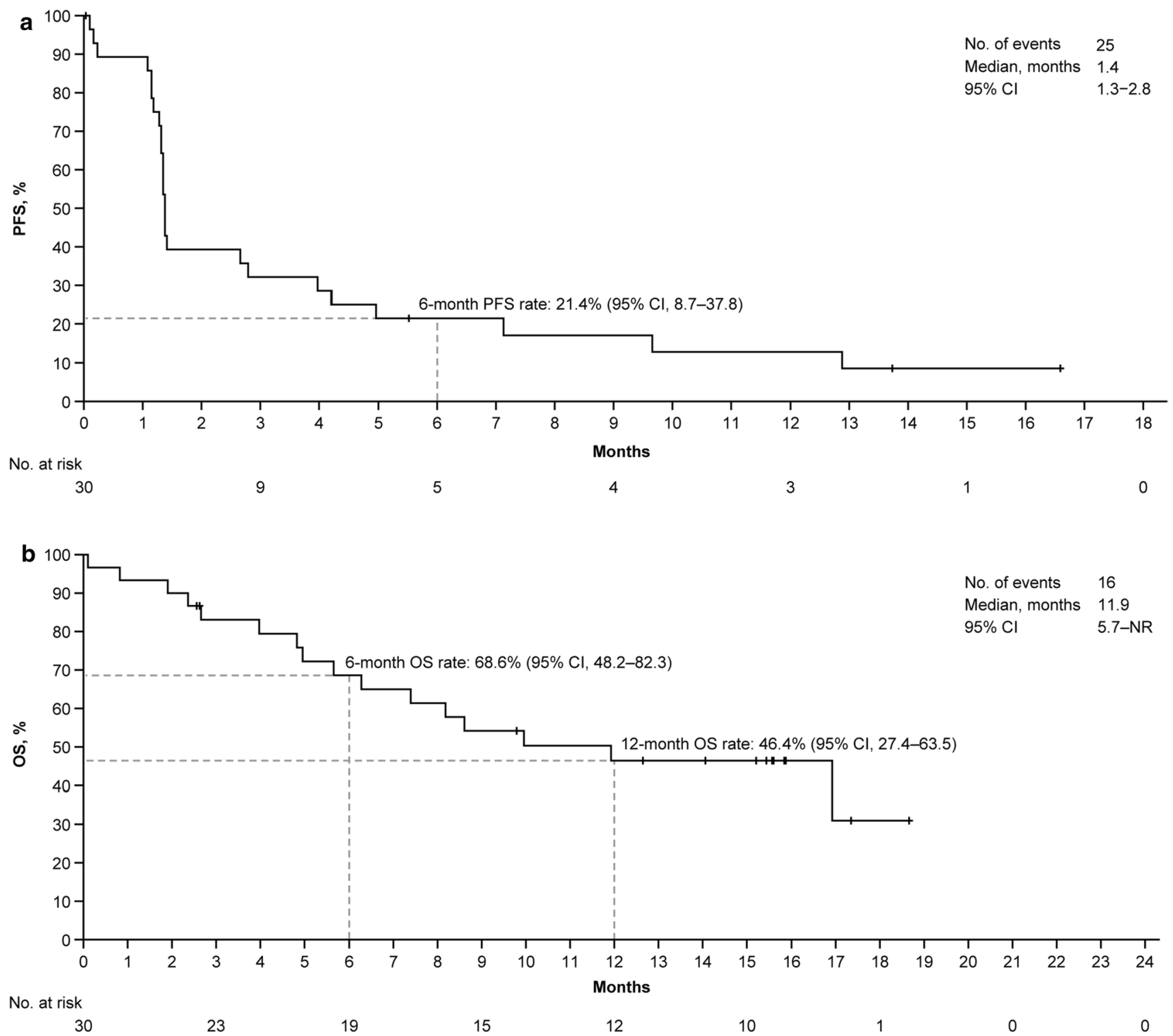

Fig. 2 Survival outcomes. Kaplan-Meier survival curves for a independent review committee-assessed PFS and b OS. CI confidence interval, $N R$ not reached, $O S$ overall survival, $P F S$ progression-free survival

mutation count or expression of genes associated with an active immune pathway (such as $C D 8 A, C D 8 B$, and $I F N G$ ) or genes commonly associated with TGF $\beta$ activation (such as TGFB1, TWIST1, and VIM) (Figs. 1a, 3b-g). The average tumor $T G F B 1$ expression level in this esophageal SCC cohort was $71.9 \%$ higher (false discovery rate-adjusted $p$ $<0.002$ ) than in an esophageal AC cohort from a separate phase 1 study of bintrafusp alfa [54]. The same trend was also observed for $T G F B 3$; however, the difference was not significant (false discovery rate-adjusted $p=0.426$ ). TGFB2 expression was $49.1 \%$ lower in patients with esophageal SCC than in those with esophageal AC (false discovery rate-adjusted $p=0.049$ ) (Supplementary Figure S2, see the electronic supplementary material). Furthermore, TGFB1 expression in these patient samples was $57.4 \%$ higher (false discovery rate-adjusted $p<0.0001)$ and TGFB2 expression was $51.8 \%$ lower (false discovery rate-adjusted $p=0.003$ ) than in other tumor cohorts tested across phase 1 trials of bintrafusp alfa; there was no significant difference in $T G F B 3$ expression (false discovery rate-adjusted $p=0.492$ ).

\section{Discussion}

Bintrafusp alfa had a manageable safety profile in this cohort of 30 Asian patients with heavily pretreated, PD-L1-unselected esophageal SCC, with low rates of grade 3/4 TRAEs and no treatment-related deaths. The safety findings reported 

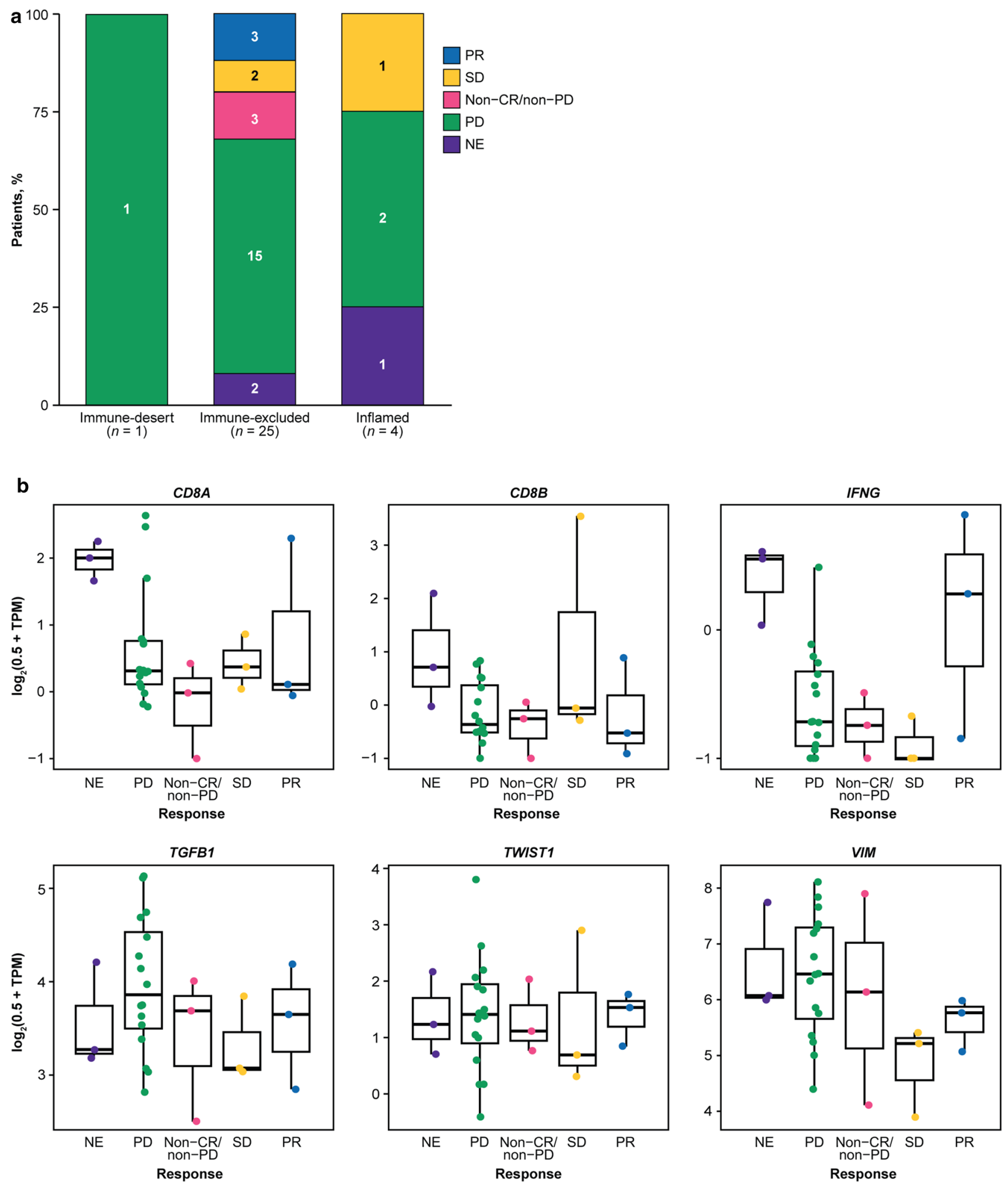

Fig. 3 Exploratory biomarker analysis by confirmed best overall response to bintrafusp alfa per independent review committee assessment. a Immune phenotype analysis. b Gene expression analysis; 2 patients with confirmed best overall responses of PD were not included in the RNAseq analysis due to failing QC. $C R$ complete response, IFNG interferon gamma, $N E$ not evaluable, $P D$ progressive disease, $P R$ partial response, $Q C$ Quality control, $R N A s e q$ RNA sequencing, $S D$ stable disease, TGFB1 transforming growth factor- $\beta$ 1, TPM transcript per million, TWIST1 twist family bHLH transcription factor 1, VIM vimentin 
here are similar to those reported with immune checkpoint inhibitors across indications, with the exception of irAEs and potentially TGF $\beta$-related skin lesions $[23,26,29,30$, 55-60]. These AEs, such as KAs, have also been observed with another TGF $\beta$-inhibiting agent [61]. In this study, potentially TGF $\beta$-related skin lesions were well managed and led to discontinuation of bintrafusp alfa in one patient. Overall, the safety profile observed for bintrafusp alfa in patients with esophageal SCC was consistent with results from an esophageal AC cohort in a separate study and other solid tumor types [38, 39, 54].

The efficacy results reported here show signs of clinical activity, with confirmed ORRs of $10.0 \%$ and $20.0 \%$ by independent review committee and investigator assessments, respectively. Furthermore, treatment with bintrafusp alfa achieved a high median OS of 11.9 months and a 12-month OS rate of $46.4 \%$ in a PD-L1-unselected population. Although direct comparisons cannot be made between trials due to key differences in study design, the median OS observed with bintrafusp alfa in this study is higher than values from large clinical trials of similar populations treated with PD-1 inhibitors (range 6.8-10.9 months) [23, 26, 29, $30,59]$. Larger studies with an active comparator are needed in order to determine the clinical benefit of bintrafusp alfa in esophageal SCC.

Responses were observed independent of PD-L1 expression, occurring in both PD-L1-positive and PD-L1-negative disease. Additional studies investigating bintrafusp alfa in a larger population would be needed to confirm whether PD-L1 expression has an impact on the efficacy of bintrafusp alfa in esophageal SCC.

Results of the exploratory analyses showed that response to bintrafusp alfa was independent of tumor mutation count. However, a higher mutation count was observed in this esophageal SCC cohort than in patients with esophageal AC from a separate phase 1 study (NCT02517398) [54]. Analysis of archival tumor samples showed significant differences in the average expression levels of $T G F B 1$ (higher) and $T G F B 2$ (lower) in this cohort compared with other tumor cohorts from phase 1 studies of bintrafusp alfa, including esophageal AC. TGF $\beta$ signaling is thought to have a role in limiting T cell infiltration in the TME and is associated with lack of response to immune checkpoint inhibitor treatment, especially in tumors with an immune-excluded phenotype $[31,62]$. In mice models of immune-excluded cancers, studies have shown a significant increase in tumor-infiltrating $\mathrm{T}$ cells and a significant reduction in tumor burden when using both a TGF $\beta$ inhibitor and PD-L1 inhibitor [31]. In this study, responses to bintrafusp alfa were only seen in tumors with an immune-excluded phenotype. Interestingly, in an esophageal AC cohort from the other phase 1 study, all but one response to bintrafusp alfa also occurred in patients with immune-excluded tumors [54]. Taken together, the results of the exploratory analyses did not identify predictive biomarkers of response to bintrafusp alfa in this small cohort.

Limitations of this study include the small number of patients and lack of a comparator arm, which preclude any definitive conclusions regarding comparisons of bintrafusp alfa with other treatments. Additionally, enrollment was restricted to Asian patients, and therefore the efficacy of bintrafusp alfa in esophageal SCC was not evaluated in other ethnic groups. Nevertheless, the results from this cohort highlight the efficacy of a novel anticancer agent in patients from eastern Asia, which has a high incidence of esophageal SCC [1].

Taken together, the safety profile of bintrafusp alfa in this study was manageable and consistent with inhibition of the TGF $\beta$ and PD-L1 pathways. Additionally, a median OS of 11.9 months was observed with bintrafusp alfa treatment in this expansion cohort. Further clinical investigation of bintrafusp alfa in esophageal cancer is warranted based on these results.

Supplementary Information The online version contains supplementary material available at https://doi.org/10.1007/s11523-021-00810-9.

Acknowledgements The authors thank the patients and their families, investigators, coinvestigators, and study teams at each of the participating centers and at Merck KGaA, Darmstadt, Germany. The authors thank Christian Ihling, of Merck KGaA, and George Locke, of EMD Serono Research \& Development Institute, Inc., Billerica, Massachusetts, USA, an affiliate of Merck KGaA, Darmstadt, Germany, for their substantial contribution to the immune phenotype and biomarker analysis, respectively. Medical writing support was provided by Lauren Rusnak, PhD, of ClinicalThinking Inc., Hamilton, NJ, USA, which was also funded by Merck KGaA and GlaxoSmithKline in accordance with Good Publication Practice (GPP3) guidelines (http://www.ismpp. org/gpp3).

\section{Declarations}

Funding This trial was funded by Merck KGaA, Darmstadt, Germany, and is part of an alliance between Merck KGaA and GlaxoSmithKline. Merck KGaA provided the study drug and worked with investigators on the trial design and plan, collection and analysis of data, and interpretation of results. Funding for a professional medical writer with access to the data was provided by Merck KGaA and GlaxoSmithKline.

Conflicts of interest C.-C. Lin reports honoraria from Eli Lilly, Novartis, and Roche; has consulted for Blueprint Medicines, Boehringer Ingelheim, Bristol Myers Squibb, Daiichi Sankyo, Eli Lilly, and Novartis; and received travel grants from BeiGene and Eli Lilly. T. Doi has consulted for Taiho, Merck Sharp \& Dohme, Amgen, Sumitomo Dainippon Pharma, Rakuten Medical, Daiichi Sankyo, Takeda, Bayer, Novartis, Boehringer Ingelheim, AbbVie, and Janssen Pharma; has received research grants from Bristol Myers Squibb, Taiho, Merck Sharp \& Dohme, Novartis, Merck Biopharma Co., Ltd., Japan—an affiliate of Merck KGaA, Darmstadt, Germany-Lilly, AbbVie, Boehringer Ingelheim, Eisai, Kyowa Hakko Kirin, IQVIA, Pfizer, Sumitomo Dainippon Pharma, and Daiichi Sankyo; and reports honoraria from Bristol Myers Squibb, Astellas, AbbVie, Ono Pharmaceutical, Oncolys BioPharma, and Taiho. K. Muro has consulted for Ono Pharmaceuti- 
cal, Amgen, and Solasia Pharma; has received research grants from Parexel International, Merck Biopharma Co., Ltd., Japan—an affiliate of Merck KGaA, Darmstadt, Germany-Merck Sharp \& Dohme, Daiichi Sankyo, Sanofi, Sumitomo Dainippon Pharma, Shionogi, Pfizer, Mediscience Planning, and Solasia Pharma; and reports honoraria from Eli Lilly, Chugai, Takeda, Ono Pharmaceutical, Taiho, Sanofi, Bristol Myers Squibb, and Bayer. T. Esaki received research grants from Merck Sharp \& Dohme, Novartis, Sumitomo Dainippon Pharma, Ono Pharmaceutical, Daiichi Sankyo, Astellas, Amgen, BeiGene, Pierre Fabre Medicament, Ignyta, Array BioPharma, Bayer, Taiho, and Lilly; and reports honoraria from Chugai, Taiho, Merck Sharp \& Dohme, Eli Lilly, Daiichi Sankyo, Nihon Kayaku, Sanofi, Ono Pharmaceutical, Takeda, Bayer, Merck Biopharma Co., Ltd., Japan-an affiliate of Merck KGaA, Darmstadt, Germany-Bristol Myers Squibb, and Eisai. H. Hara has consulted for Lilly, Merck Sharp \& Dohme, and Ono Pharmaceutical; received research grants from Astellas, AstraZeneca, BeiGene, Boehringer Ingelheim, Chugai, Daiichi Sankyo, Sumitomo Dainippon Pharma, Eisai, Incyte, LSK BioPharma, Merck Biopharma Co., Ltd., Japan—an affiliate of Merck KGaA, Darmstadt, Germany-Merck Sharp \& Dohme, Ono Pharmaceutical, Pfizer, and Taiho; and reports honoraria from Bayer, Bristol Myers Squibb, Chugai, Daiichi Sankyo, Kyowa Hakko Kirin, Lilly, Merck Biopharma Co., Ltd., Japan - an affiliate of Merck KGaA, Darmstadt, GermanyMerck Sharp \& Dohme, Ono Pharmaceutical, Sanofi, Taiho, Takeda, and Yakult. C. Helwig is an employee of and has ownership interest in Merck KGaA, Darmstadt, Germany. I. Dussault is an employee of EMD Serono Research \& Development Institute, Inc., Billerica, MA, USA - an affiliate of Merck KGaA, Darmstadt, Germany-and has ownership interest in EMD Serono Research \& Development Institute, Inc., and Amgen. M. Osada is an employee of Merck Biopharma Co., Ltd., Japan - an affiliate of Merck KGaA, Darmstadt, Germany. S. Kondo has received research funding from ASLAN Pharmaceuticals, AstraZeneca, Bayer, Eli Lilly, Merck Sharp \& Dohme, and Pfizer. The remaining authors declare no competing interests.

Ethics approval and consent to participate The protocol for this study was approved by all relevant regulatory authorities and ethics committees at participating institutions, and the study was conducted in accordance with the International Conference on Harmonisation Topic E6 Good Clinical Practice and the Declaration of Helsinki. Each patient provided written informed consent before study enrollment.

Consent for publication All authors gave final approval of the version to be published.

Data availability For all new products or new indications approved in both the European Union and the United States after January 1, 2014, Merck KGaA, Darmstadt, Germany will share patient-level and study-level data after deidentification, as well as redacted study protocols and clinical study reports from clinical trials in patients. Any requests for these data by qualified scientific and medical researchers for legitimate research purposes will be subject to Merck KGaA's Data Sharing Policy. All requests should be submitted in writing to Merck KGaA's data sharing portal (https://www.merckgroup.com/en/research/ our-approach-to-research-and-development/healthcare/clinical-trials/ commitment-responsible-data-sharing.html). When Merck KGaA has a co-research, co-development, or co-marketing or co-promotion agreement, or when the product has been out-licensed, the responsibility for disclosure might be dependent on the agreement between parties. Under these circumstances, Merck KGaA will endeavor to gain agreement to share data in response to requests.

Authors' contributions $\mathrm{CH}$, ID, and $\mathrm{MO}$ contributed to study conception and design; CCL, TD, KM, M-MH, TE, HH, HCC, and SK contributed to data acquisition. All authors contributed to data interpreta- tion and drafting of the manuscript. All authors reviewed and approved the final manuscript and approved the final manuscript for publication.

Open Access This article is licensed under a Creative Commons Attribution-NonCommercial 4.0 International License, which permits any non-commercial use, sharing, adaptation, distribution and reproduction in any medium or format, as long as you give appropriate credit to the original author(s) and the source, provide a link to the Creative Commons licence, and indicate if changes were made. The images or other third party material in this article are included in the article's Creative Commons licence, unless indicated otherwise in a credit line to the material. If material is not included in the article's Creative Commons licence and your intended use is not permitted by statutory regulation or exceeds the permitted use, you will need to obtain permission directly from the copyright holder. To view a copy of this licence, visit $\mathrm{http} / / /$ creativecommons.org/licenses/by-nc/4.0/.

\section{References}

1. Bray F, Ferlay J, Soerjomataram I, Siegel RL, Torre LA, Jemal A. Global cancer statistics 2018: GLOBOCAN estimates of incidence and mortality worldwide for 36 cancers in 185 countries. CA Cancer J Clin. 2018;68(6):394-424.

2. Allemani C, Matsuda T, Di Carlo V, Harewood R, Matz M, Nikšić M, et al. Global surveillance of trends in cancer survival 2000-14 (CONCORD-3): analysis of individual records for 37513025 patients diagnosed with one of 18 cancers from 322 population-based registries in 71 countries. Lancet. 2018;391(10125):1023-75.

3. Zhang Y. Epidemiology of esophageal cancer. World J Gastroenterol. 2013;19(34):5598-606.

4. Abnet CC, Arnold M, Wei W-Q. Epidemiology of esophageal squamous cell carcinoma. Gastroenterology. 2018;154(2):360-73. https://doi.org/10.1053/j.gastro.2017.08.023.

5. Lu C-L, Lang H-C, Luo J-C, Liu C-C, Lin H-C, Chang F-Y, et al. Increasing trend of the incidence of esophageal squamous cell carcinoma, but not adenocarcinoma, in Taiwan. Cancer Causes Control. 2010;21(2):269-74. https://doi.org/10.1007/ s10552-009-9458-0.

6. Arnold M, Laversanne M, Brown LM, Devesa SS, Bray F. Predicting the future burden of esophageal cancer by histological subtype: international trends in incidence up to 2030. Gastroenterology. 2017;112(8):1247-55.

7. Yao PF, Li GC, Li J, Xia HS, Yang XL, Huang HY, et al. Evidence of human papilloma virus infection and its epidemiology in esophageal squamous cell carcinoma. World J Gastroenterol. 2006;12(9):1352-5. https://doi.org/10.3748/wjg.v12.i9.1352.

8. The Cancer Genome Atlas Research Network, Kim J, Bowlby R, Mungall AJ, Robertson AG, Odze RD, et al. Integrated genomic characterization of oesophageal carcinoma. Nature. 2017;541:169-75.

9. Lin EW, Karakasheva TA, Hicks PD, Bass AJ, Rustgi AK. The tumor microenvironment in esophageal cancer. Oncogene. 2016;35:5337.

10. Akhurst RJ, Hata A. Targeting the TGF $\beta$ signalling pathway in disease. Nat Rev Drug Discov. 2012;11(10):790-811.

11. Principe DR, Doll JA, Bauer J, Jung B, Munshi HG, Bartholin L, et al. TGF- $\beta$ : duality of function between tumor prevention and carcinogenesis. J Natl Cancer Inst. 2014;106(2):djt369.

12. Fukai Y, Fukuchi M, Masuda N, Osawa H, Kato H, Nakajima T, et al. Reduced expression of transforming growth factor- $\beta$ receptors is an unfavorable prognostic factor in human esophageal squamous cell carcinoma. Int J Cancer. 2003;104(2):161-6. 
13. Peng L-s, Zhang J-y, Teng Y-s, Zhao Y-l, Wang T-t, Mao F-y, et al. Tumor-associated monocytes/macrophages impair NK-cell function via TGF $\beta 1$ in human gastric cancer. Cancer Immunol Res. 2017;5(3):248-56. https://doi.org/10.1158/2326-6066. Cir-16-0152.

14. Sun SP, Jin YN, Yang HP, Wei Y, Dong Z. Serum transforming growth factor-beta1 level reflects disease status in patients with esophageal carcinoma after radiotherapy. World J Gastroenterol. 2007;13(39):5267-72. https://doi.org/10.3748/wjg.v13.i39.5267.

15. Gholamin M, Moaven O, Memar B, Farshchian M, Naseh H, Malekzadeh R, et al. Overexpression and interactions of interleukin-10, transforming growth factor $\beta$, and vascular endothelial growth factor in esophageal squamous cell carcinoma. World J Surg. 2009;33(7):1439.

16. Colak S, ten Dijke P. Targeting TGF- $\beta$ signaling in cancer. Trends Cancer. 2017;3(1):56-71. https://doi.org/10.1016/j.trecan.2016. 11.008 .

17. Pang L, Li Q, Wei C, Zou H, Li S, Cao W, et al. TGF- $\beta 1 /$ Smad signaling pathway regulates epithelial-to-mesenchymal transition in esophageal squamous cell carcinoma: in vitro and clinical analyses of cell lines and nomadic Kazakh patients from northwest Xinjiang, China. PLoS ONE. 2014;9(12):e112300. https://doi.org/ 10.1371/journal.pone.0112300.

18. Muro K, Lordick F, Tsushima T, Pentheroudakis G, Baba E, Lu $\mathrm{Z}$, et al. Pan-Asian adapted ESMO clinical practice guidelines for the management of patients with metastatic oesophageal cancer: a JSMO-ESMO initiative endorsed by CSCO, KSMO, MOS, SSO and TOS. Ann Oncol. 2019;30(1):34-43. https://doi.org/10.1093/ annonc/mdy498.

19. Lordick F, Mariette C, Haustermans K, Obermannová R, Arnold D. Oesophageal cancer: ESMO clinical practice guidelines for diagnosis, treatment and follow-up. Ann Oncol. 2016;27(suppl 5):v50-7. https://doi.org/10.1093/annonc/mdw329.

20. National Comprehensive Cancer N. NCCN Clinical Practice Guidelines in Oncology (NCCN guidelines): esophageal and esophagogastric junction cancers, V.2.2019. 2019.

21. Ter Veer E, Haj Mohammad N, van Valkenhoef G, Ngai LL, Mali RMA, van Oijen MGH, et al. Second- and third-line systemic therapy in patients with advanced esophagogastric cancer: a systematic review of the literature. Cancer Metastasis Rev. 2016;35(3):439-56. https://doi.org/10.1007/s10555-016-9632-2.

22. Song Z, Zhang Y. Second-line docetaxel-based chemotherapy after failure of fluorouracil-based first-line treatment for advanced esophageal squamous cell carcinoma. Onco Targets Ther. 2014;7:1875-81. https://doi.org/10.2147/ott.S66525.

23. Kojima T, Shah MA, Muro K, Francois E, Adenis A, Hsu C-H, et al. Randomized phase III KEYNOTE-181 study of pembrolizumab versus chemotherapy in advanced esophageal cancer. J Clin Oncol. 2020;38(35):4138-48.

24. Merck. News release. Merck's KEYTRUDA® (pembrolizumab) approved in China for second-line treatment of patients with locally advanced or metastatic esophageal squamous cell carcinoma whose tumors express PD-L1 (CPS $\geq 10$ ). 2020. https:// www.merck.com/news/mercks-keytruda-pembrolizumab-appro ved-in-china-for-second-line-treatment-of-patients-with-locallyadvanced-or-metastatic-esophageal-squamous-cell-carcinomawhose-tumors-express-pd-11. Accessed 26 Feb 2021.

25. Administration USFaD. FDA approves pembrolizumab for advanced esophageal squamous cell cancer. 2019. https://www. fda.gov/drugs/resources-information-approved-drugs/fda-appro ves-pembrolizumab-advanced-esophageal-squamous-cell-cancer

26. Shah MA, Kojima T, Hochhauser D, Enzinger P, Raimbourg J, Hollebecque A, et al. Efficacy and safety of pembrolizumab for heavily pretreated patients with advanced, metastatic adenocarcinoma or squamous cell carcinoma of the esophagus: the phase 2 KEYNOTE-180 study. JAMA Oncol. 2019;5(4):546-50.
27. Kato K, Sun J, Shah MA, Enzinger PC, Adenis A, Doi T, et al. Pembrolizumab plus chemotherapy versus chemotherapy as firstline therapy in patients with advanced esophageal cancer: the phase 3 KEYNOTE-590 study. Ann Oncol. 2020;31(suppl 4): LBA8_PR.

28. Merck. News release. FDA grants priority review to Merck's supplemental biologics license application for KEYTRUDA (pembrolizumab) plus chemotherapy as first-line treatment for locally advanced unresectable or metastatic esophageal and gastroesophageal junction cancer. 2020. https://www.merck.com/ news/fda-grants-priority-review-to-mercks-supplemental-biolo gics-license-application-for-keytruda-pembrolizumab-plus-chemo therapy-as-first-line-treatment-for-locally-advanced-unresectable. Accessed 26 Feb 2021.

29. Kato K, Cho BC, Takahashi M, Okada M, Lin C-Y, Chin K, et al. Nivolumab versus chemotherapy in patients with advanced oesophageal squamous cell carcinoma refractory or intolerant to previous chemotherapy (ATTRACTION-3): a multicentre, randomised, open-label, phase 3 trial. Lancet Oncol. 2019;20(11):1506-17. https://doi.org/10.1016/S1470-2045(19) 30626-6.

30. Kudo T, Hamamoto Y, Kato K, Ura T, Kojima T, Tsushima T, et al. Nivolumab treatment for oesophageal squamous-cell carcinoma: an open-label, multicentre, phase 2 trial. Lancet Oncol. 2017;18(5):631-9. https://doi.org/10.1016/S1470-2045(17) 30181-X.

31. Mariathasan S, Turley SJ, Nickles D, Castiglioni A, Yuen K, Wang Y, et al. TGF $\beta$ attenuates tumour response to PD-L1 blockade by contributing to exclusion of T cells. Nature. 2018;554:544-8.

32. Holmgaard RB, Schaer DA, Li Y, Castaneda SP, Murphy MY, $\mathrm{Xu} \mathrm{X}$, et al. Targeting the TGF $\beta$ pathway with galunisertib, a TGF $\beta R I$ small molecule inhibitor, promotes anti-tumor immunity leading to durable, complete responses, as monotherapy and in combination with checkpoint blockade. J ImmunoTher Cancer. 2018;6(1):47.

33. Tauriello DVF, Palomo-Ponce S, Stork D, Berenguer-Llergo A, Badia-Ramentol J, Iglesias M, et al. TGF $\beta$ drives immune evasion in genetically reconstituted colon cancer metastasis. Nature. 2018;554:538-43.

34. Principe DR, Park A, Dorman MJ, Kumar S, Viswakarma N, Rubin J, et al. TGF $\beta$ blockade augments PD-1 inhibition to promote T-cell-mediated regression of pancreatic cancer. Mol Cancer Ther. 2019;18(3):613-20.

35. Lan Y, Zhang D, Xu C, Hance KW, Marelli B, Qi J, et al. Enhanced preclinical antitumor activity of M7824, a bifunctional fusion protein simultaneously targeting PD-L1 and TGF- $\beta$. Sci Transl Med. 2018;10(424):eaan5488.

36. Knudson KM, Hicks KC, Luo X, Chen JQ, Schlom J, Gameiro SR. M7824, a novel bifunctional anti-PD-L1/TGF $\beta$ Trap fusion protein, promotes anti-tumor efficacy as monotherapy and in combination with vaccine. Oncoimmunology. 2018;7(5):e1426519.

37. David JM, Dominguez C, McCampbell KK, Gulley JL, Schlom J, Palena C. A novel bifunctional anti-PD-L1/TGF- $\beta$ Trap fusion protein (M7824) efficiently reverts mesenchymalization of human lung cancer cells. Oncoimmunology. 2017;6(10):e1349589.

38. Strauss J, Heery CR, Schlom J, Madan RA, Cao L, Kang Z, et al. Phase I trial of M7824 (MSB0011359C), a bifunctional fusion protein targeting PD-L1 and TGF $\beta$, in advanced solid tumors. Clin Cancer Res. 2018;24(6):1287-95.

39. Doi T, Fujiwara Y, Koyama T, Ikeda M, Helwig C, Watanabe M, et al. Phase I study of the bifunctional fusion protein bintrafusp alfa in Asian patients with advanced solid tumors, including a hepatocellular carcinoma safety-assessment cohort. Oncologist. 2020;25(9):e1292-302.

40. Lin CC, Doi T, Muro K, Hou MM, Esaki T, Hara H, et al. Phase I study results from an esophageal squamous cell carcinoma 
(ESCC) cohort treated withM7824 (MSB0011359C), a bifunctional fusion protein targeting transforming growth factor $\beta$ (TGF$\beta$ ) and PD-L1. Ann Oncol. 2018;29:642P. https://doi.org/10.1093/ annonc/mdy282.026.

41. Vugmeyster Y, Wilkins J, Koenig A, El Bawab S, Dussault I, Ojalvo LS, et al. Selection of the recommended phase 2 dose for bintrafusp alfa, a bifunctional fusion protein targeting TGF- $\beta$ and PD-L1. Clin Pharmacol Ther. 2020;108(3):566-74.

42. Wilkins JJ, Vugmeyster Y, Dussault I, Girard P, Khandelwal A. Population pharmacokinetic analysis of bintrafusp alfa in different cancer types. Adv Ther. 2019;36(9):2414-33. https://doi.org/10. 1007/s12325-019-01018-0.

43. Langmead B, Salzberg SL. Fast gapped-read alignment with Bowtie 2. Nat Methods. 2012;9(4):357-9.

44. Li B, Dewey CN. RSEM: accurate transcript quantification from RNA-Seq data with or without a reference genome. BMC Bioinform. 2011;12(1):323.

45. Ritchie ME, Phipson B, Wu D, Hu Y, Law CW, Shi W, et al. limma powers differential expression analyses for RNA-sequencing and microarray studies. Nucleic Acids Res. 2015;43(7):e47.

46. Dobin A, Davis CA, Schlesinger F, Drenkow J, Zaleski C, Jha S, et al. STAR: ultrafast universal RNA-seq aligner. Bioinformatics. 2012;29(1):15-21.

47. Li H, Durbin R. Fast and accurate short read alignment with Burrows-Wheeler transform. Bioinformatics. 2009;25(14):1754-60.

48. Lai Z, Markovets A, Ahdesmaki M, Chapman B, Hofmann O, McEwen R, et al. VarDict: a novel and versatile variant caller for next-generation sequencing in cancer research. Nucleic Acids Res. 2016;44(11):e108. https://doi.org/10.1093/nar/gkw227.

49. McLaren W, Gil L, Hunt SE, Riat HS, Ritchie GR, Thormann A, et al. The Ensembl variant effect predictor. Genome Biol. 2016;17(1):122.

50. Chen DS, Mellman I. Elements of cancer immunity and the cancer-immune set point. Nature. 2017;541:321-30.

51. Kim JM, Chen DS. Immune escape to PD-L1/PD-1 blockade: seven steps to success (or failure). Ann Oncol. 2016;27(8):1492-504.

52. Hegde PS, Karanikas V, Evers S. The where, the when, and the how of immune monitoring for cancer immunotherapies in the era of checkpoint inhibition. Clin Cancer Res. 2016;22(8):1865-74.

53. Bacac M, Fauti T, Sam J, Colombetti S, Weinzierl T, Ouaret D, et al. A novel carcinoembryonic antigen T-cell bispecific antibody
(CEA TCB) for the treatment of solid tumors. Clin Cancer Res. 2016;22(13):3286-97. https://doi.org/10.1158/1078-0432. ccr-15-1696.

54. Tan B, Khattak A, Felip E, Kelly K, Rich P, Wang D, et al. Bintrafusp alfa, a bifunctional fusion protein targeting TGF- $\beta$ and PD-L1, in patients with esophageal adenocarcinoma: results from a phase 1 cohort. Target Oncol. 2021. https://doi.org/10.1007/ s11523-021-00809-2

55. Ralph C, Elkord E, Burt DJ, O'Dwyer JF, Austin EB, Stern PL, et al. Modulation of lymphocyte regulation for cancer therapy: a phase II trial of tremelimumab in advanced gastric and esophageal adenocarcinoma. Clin Cancer Res. 2010;16(5):1662-72.

56. Doi T, Piha-Paul SA, Jalal SI, Saraf S, Lunceford J, Koshiji $\mathrm{M}$, et al. Safety and antitumor activity of the anti-programmed death-1 antibody pembrolizumab in patients with advanced esophageal carcinoma. J Clin Oncol. 2018;36(1):61-7.

57. Shen J, Chang J, Mendenhall M, Cherry G, Goldman JW, Kulkarni RP. Diverse cutaneous adverse eruptions caused by anti-programmed cell death-1 (PD-1) and anti-programmed cell death ligand-1 (PD-L1) immunotherapies: clinical features and management. Ther Adv Med Oncol. 2018;10:1758834017751634.

58. Khoja L, Day D, Wei-Wu Chen T, Siu LL, Hansen AR. Tumourand class-specific patterns of immune-related adverse events of immune checkpoint inhibitors: a systematic review. Ann Oncol. 2017;28(10):2377-85.

59. Kojima T, Muro K, Francois E, Hsu C-H, Moriwaki T, Kim S-B, et al. Pembrolizumab versus chemotherapy as second-line therapy for advanced esophageal cancer: phase III KEYNOTE-181 study. J Clin Oncol. 2019;37(4 suppl):2. https://doi.org/10.1200/JCO. 2019.37.4_suppl.2.

60. Fuchs CS, Doi T, Jang RW, Muro K, Satoh T, Machado M, et al. Safety and efficacy of pembrolizumab monotherapy in patients with previously treated advanced gastric and gastroesophageal junction cancer: phase 2 clinical KEYNOTE-059 trial. JAMA Oncol. 2018;4:e180013.

61. Lacouture ME, Morris JC, Lawrence DP, Tan AR, Olencki TE, Shapiro GI, et al. Cutaneous keratoacanthomas/squamous cell carcinomas associated with neutralization of transforming growth factor $\beta$ by the monoclonal antibody fresolimumab (GC1008). Cancer Immunol Immunother. 2015;64(4):437-46.

62. Hegde PS, Chen DS. Top 10 challenges in cancer immunotherapy. Immunity. 2020;52(1):17-35.

\section{Authors and Affiliations}

\section{Chia-Chi Lin ${ }^{1} \cdot$ Toshihiko Doi $^{2} \cdot$ Kei Muro $^{3} \cdot$ Ming-Mo Hou $^{4} \cdot$ Taito Esaki $^{5} \cdot$ Hiroki Hara $^{6} \cdot \mathrm{Hyun}_{\text {Cheol Chung }}$. Christoph Helwig $^{8} \cdot$ Isabelle Dussault $^{9,10} \cdot$ Motonobu Osada ${ }^{11,12} \cdot$ Shunsuke Kondo ${ }^{13}$}

1 National Taiwan University Hospital, Taipei, Taiwan

2 National Cancer Center Hospital East, Kashiwa, Japan

3 Aichi Cancer Center Hospital, Nagoya, Japan

4 Chang Gung Memorial Hospital and Chang Gung University, Taoyuan, Taiwan

5 National Hospital Organization Kyushu Cancer Center, Fukuoka, Japan

6 Saitama Cancer Center, Saitama, Japan

7 Yonsei Cancer Center, Yonsei University College of Medicine, Seoul, Korea

8 Merck KGaA, Darmstadt, Germany
9 EMD Serono Research and Development Institute, Inc., Billerica, MA, USA

10 An Affiliate of Merck KGaA, Darmstadt, Germany

11 Merck Biopharma Co., Ltd., Tokyo, Japan

12 An Affiliate of Merck KGaA, Darmstadt, Germany

13 National Cancer Center Hospital, 5-1-1 Tsukiji, Chuo-ku, Tokyo 104-0045, Japan 